\title{
КОРРЕКТНОСТЬ ЗАДАЧИ ДИРИХЛЕ В ЦИЛИНДРИЧЕСКОЙ ОБЛАСТИ ДЛЯ ВЫРОЖДАЮЩИХСЯ МНОГОМЕРНЫХ ЭЛЛИПТИКО-ПАРАБОЛИЧЕСКИХ УРАВНЕНИЙ
}

\begin{abstract}
Корректность краевых задач на плоскости для эллиптических уравнений методом теории аналитических функций комплексного переменного хорошо изучена. При исследовании аналогичных вопросов, когда число независимых переменных больше двух, возникают трудности принципиального характера. Весьма привлекательный и удобный метод сингулярных интегральных уравнений теряет свою силу из-за отсутствия сколько-нибудь полной теории многомерных сингулярных интегральных уравнений. Краевые задачи для эллиптических уравнений второго порядка в областях с ребрами подробно изучены.

В работах автора найдены явные виды классических решений задач Дирихле в цилиндрических областях для многомерных эллиптических уравнений. В данной статье используется метод, предложенный в работах автора, показана однозначная разрешимость и получен явный вид классического решения задачи Дирихле в цилиндрической области для одного класса вырождающихся многомерных эллиптико-параболических уравнений.
\end{abstract}

Ключевые слова: корректность, многомерные уравнения, вырождение, задача Дирихле, цилиндрическая область, сферические функции, ортогональность, функция Бесселя.

Для общих эллиптико-параболических уравнений второго порядка постановку первой краевой задачи (или задача Дирихле) впервые осуществил Г. Фикера [1]. Дальнейшее изучение этой задачи приведено в $[2]$.

В работе для вырождающихся многомерных эллиптико-параболических уравнений доказано однозначная разрешимость и получен явный вид классического решения задачи Дирихле в цилиндрической области.

Пусть $\Omega_{\alpha \beta}$ - цилиндрическая область евклидова пространства $E_{m+1}$ точек $\left(x_{1}, \ldots, x_{m}, t\right)$, ограниченная цилиндром $\Gamma=\{(x, t):|x|=1\}$, плоскостями $t=\alpha>0$ и $t=\beta<0$, где $|x|$ - длина вектора $x=\left(x_{1}, \ldots, x_{m}\right)$.

Обозначим через $\Omega_{\alpha}$ и $\Omega_{\beta}$ части области $\Omega_{\alpha \beta}$, а через $\Gamma_{\alpha}, \Gamma_{\beta}-$ части поверхности $\Gamma$, лежащие в полупространствах $t>0$ и $t<0 ; \sigma_{\alpha}$ - верхнее, $\sigma_{\beta}$ - нижнее основание области $\Omega_{\alpha \beta}$.

Пусть далее $S$ - общая часть границ областей $\Omega_{\alpha}, \Omega_{\beta}$ представляющее множество $\{t=0,0<|x|<1\}$ B $E_{m}$.

В области $\Omega_{\alpha \beta}$ рассмотрим вырождающихся смешанно эллиптико-параболические уравнения

$$
0=\left\{\begin{array}{c}
g(t) \Delta_{x} u-u_{t}, t>0, \\
p(t) \Delta_{x} u+u_{t t}, t<0,
\end{array}\right.
$$

где $g(t)>0$ при $t>0$, и может обращаться в нули при $t=0, g(t) \in C([0, \alpha]), p(t)>0$ при $t<0$, и может обращаться в нуль при $t=0, p(t) \in C([\beta, 0])$, а $\Delta_{x}$ - оператор Лапласа по переменным $x_{1}, \ldots, x_{m}, m \geqslant 2$.

В дальнейшем нам удобно перейти от декартовых координат $x_{1}, \ldots, x_{m}, t$ к сферическим $r, \theta_{1}, \ldots, \theta_{m-1}, t, r \geqslant 0,0 \leqslant \theta_{1}<2 \pi, 0 \leqslant \theta_{i} \leqslant \pi, i=2,3, \ldots, m-1, \theta=\left(\theta_{1}, \ldots, \theta_{m-1}\right)$.

Задача 1 (Дирихле). Найти решение уравнения (1) в области $\Omega_{\alpha \beta}$ при $t \neq 0$ из класса $C\left(\bar{\Omega}_{\alpha \beta}\right) \cap$ $\cap C^{2}\left(\Omega_{\alpha} \cup \Omega_{\beta}\right)$, удовлетворяющее краевым условиям

$$
\begin{gathered}
\left.u\right|_{\sigma_{\alpha}}=\varphi_{1}(r, \theta),\left.\quad u\right|_{\Gamma_{\alpha}}=\psi_{1}(t, \theta), \\
\left.u\right|_{\Gamma_{\beta}}=\psi_{2}(t, \theta),\left.u\right|_{\sigma_{\beta}}=\varphi_{2}(t, \theta) .
\end{gathered}
$$

\footnotetext{
${ }^{1}$ (C) Алдашев С.А., 2017
}

Алдашев Серик Аймурзаевич (aldash51@mail.ru), кафедра математики и математического моделирования, Институт математики и математического моделирования KН MOH PK, 050010, Республика Казахстан, г. Алматы, ул. Пушкина 125. 
при этом $\varphi_{1}(1, \theta)=\psi_{1}(\alpha, \theta), \varphi_{2}(1, \theta)=\psi_{2}(\beta, \theta), \psi_{1}(0, \theta)=\psi_{2}(0, \theta)$.

Пусть $\left\{Y_{n, m}^{k}(\theta)\right\}$ - система линейно независимых сферических функций порядка $n, 1 \leqslant k \leqslant k_{n}$, $(m-2) ! n ! k_{n}=(n+m-3) !(2 n+m-2), W_{2}^{l}(S), l=0,1, \ldots$ - пространства Соболева.

Имеет место ([3])

Лемма 1. Пусть $f(r, \theta) \in W_{2}^{l}(S)$. Если $l \geqslant m-1$, то ряд

$$
f(r, \theta)=\sum_{n=0}^{\infty} \sum_{k=1}^{k_{n}} f_{n}^{k}(r) Y_{n, m}^{k}(\theta)
$$

а также ряды, полученные из него дифференцированием порядка $p \leqslant l-m+1$, сходятся абсолютно и равномерно.

Лемма 2. Для того, чтобы $f(r, \theta) \in W_{2}^{l}(S)$, необходимо и достаточно, чтобы коэффициенты ряда $(4)$ удовлетворяли неравенствам

$$
\left|f_{0}^{1}(r)\right| \leqslant c_{1}, \sum_{n=1}^{\infty} \sum_{k=1}^{k_{n}} n^{2 l}\left|f_{n}^{k}(r)\right|^{2} \leqslant c_{2}, \quad c_{1}, c_{2}=\text { const. }
$$

Через $\bar{\varphi}_{1 n}^{k}(r), \bar{\varphi}_{2 n}^{k}(r), \psi_{1 n}^{k}(t), \psi_{2 n}^{k}(t)$, обозначим коэффициенты разложения ряда (4), соответственно функций $\varphi_{1}(r, \theta), \varphi_{2}(r, \theta), \psi_{1}(t, \theta), \psi_{2}(t, \theta)$.

Тогда справедлива

Теорема. Если $\varphi_{1}(r, \theta), \varphi_{2}(r, \theta) \in W_{2}^{l}(S), \psi_{1}(t, \theta) \in W_{2}^{l}\left(\Gamma_{\alpha}\right), \psi_{2}(t, \theta) \in W_{2}^{l}\left(\Gamma_{\beta}\right)$, $l>\frac{3 m}{2}$, то задача 1 однозначно разрешима.

Доказательства теоремы. В сферических координатах уравнение (1) в области $\Omega_{\alpha}$ имеет вид

$$
\begin{gathered}
g(t)\left(u_{r r}+\frac{m-1}{r} u_{r}-\frac{1}{r^{2}} \delta u\right)-u_{t}=0, \\
\delta \equiv-\sum_{j=1}^{m-1} \frac{1}{g_{j} \sin ^{m-j-1} \theta_{j}} \frac{\partial}{\partial \theta_{j}}\left(\sin ^{m-j-1} \frac{\partial}{\partial \theta_{j}}\right), g_{1}=1, g_{j}=\left(\sin \theta_{1} \ldots \sin \theta_{j-1}\right)^{2}, j>1 .
\end{gathered}
$$

Известно ([3]), что спектр оператора $\delta$ состоит из собственных чисел $\lambda_{n}=n(n+m-2), \quad n=0,1, \ldots$, каждому из которых соответствует $k_{n}$ ортонормированных собственных фукций $Y_{n, m}^{k}(\theta)$.

Так как искомое решение задачи 1 в области $\Omega_{\alpha}$ принадлежит классу $C\left(\bar{\Omega}_{\alpha}\right) \cap C^{2}\left(\Omega_{\alpha}\right)$, то его можно искать в виде

$$
u(r, \theta, t)=\sum_{n=0}^{\infty} \sum_{k=1}^{k_{n}} \bar{u}_{n}^{k}(r, t) Y_{n, m}^{k}(\theta)
$$

где $\bar{u}_{n}^{k}(r, t)$ - функции, подлежащие определению.

Подставляя (6) в (5), используя ортогональность сферических функций $Y_{n, m}^{k}(\theta)([3])$, будем иметь

$$
g(t)\left(\bar{u}_{n r r}^{k}+\frac{m-1}{r} \bar{u}_{n r}^{k}-\frac{\lambda_{n}}{r^{2}} \bar{u}_{n}^{k}\right)-\bar{u}_{n t}^{k}=0, k=\overline{1, k_{n}}, n=0,1, \ldots,
$$

при этом краевое условие (2), с учетом леммы 1 , соответственно запишется в виде

$$
\bar{u}_{n}^{k}(r, \alpha)=\bar{\varphi}_{1 n}^{k}(r), \quad \bar{u}_{n}^{k}(1, t)=\psi_{1 n}^{k}(t), \quad k=\overline{1, k_{n}}, \quad n=0,1, \ldots .
$$

В (7), (8) произведя замену переменных $\bar{v}_{n}^{k}(r, t)=\bar{u}_{n}^{k}(r, t)-\psi_{1 n}^{k}(t)$ получим

$$
\begin{gathered}
g(t)\left(\bar{v}_{n r r}^{k}+\frac{m-1}{r} \bar{v}_{n r}^{k}-\frac{\lambda_{n}}{r^{2}} \bar{v}_{n}^{k}\right)-\bar{v}_{n t}^{k}=\bar{f}_{n}^{k}(r, t), \\
\bar{v}_{n}^{k}(r, \alpha)=\varphi_{1 n}^{k}(r), \quad \bar{v}_{n}^{k}(1, t)=0, k=\overline{1, k_{n}}, \quad n=0,1, \ldots, \\
\bar{f}_{n}^{k}(r, t)=\psi_{1 n t}^{k}+\frac{\lambda_{n} g(t)}{r^{2}} \psi_{1 n}^{k}(t), \varphi_{1 n}^{k}(r)=\bar{\varphi}_{1 n}^{k}(r)-\psi_{1 n}^{k}(\alpha) .
\end{gathered}
$$

Произведя замену $\bar{v}_{n}^{k}(r, t)=r^{\frac{(1-m)}{2}} v_{n}^{k}(r, t)$ задачу (9), (10) приведем к следующей задаче

$$
\begin{gathered}
L v_{n}^{k} \equiv g(t)\left(v_{n r r}^{k}+\frac{\bar{\lambda}_{n}}{r^{2}} v_{n}^{k}\right)-v_{n t}^{k}=f_{n}^{k}(r, t), \\
v_{n}^{k}(r, \alpha)=\widetilde{\varphi}_{1 n}^{k}(r), v_{n}^{k}(1, t)=0, \\
\bar{\lambda}_{n}=\frac{\left((m-1)(3-m)-4 \lambda_{n}\right)}{4}, f_{n}^{k}(r, t)=r^{\frac{(1-m)}{2}} \bar{f}_{n}^{k}(r, t), \widetilde{\varphi}_{1 n}^{k}(r)=r^{\frac{(1-m)}{2}} \varphi_{1 n}^{k}(r) .
\end{gathered}
$$


Решение задачи (11), (12) ищем в виде $v_{n}^{k}(r, t)=v_{1 n}^{k} r, t+v_{2 n}^{k}(r, t)$, где $v_{1 n}^{k}(r, t)$ - решение задачи

$$
\begin{gathered}
L v_{1 n}^{k}=f_{n}^{k}(r, t), \\
v_{1 n}^{k}(r, \alpha)=0, v_{1 n}^{k}(1, t)=0,
\end{gathered}
$$

а $v_{2 n}^{k}(r, t)$ - решение задачи

$$
\begin{gathered}
L v_{2 n}^{k}=0, \\
v_{2 n}^{k}(r, \alpha)=\widetilde{\varphi}_{1 n}^{k}(r), v_{2 n}^{k}(1, t)=0 .
\end{gathered}
$$

Решение выше указанных задач, рассмотрим в виде

$$
v_{n}^{k}(r, t)=\sum_{s=1}^{\infty} R_{s}(r) T_{s}(t)
$$

при этом пусть

$$
f_{n}^{k}(r, t)=\sum_{s=1}^{\infty} a_{n s}^{k}(t) R_{s}(r), \tilde{\varphi}_{1 n}^{k}(r)=\sum_{s=1}^{\infty} b_{n s}^{k} R_{s}(r) .
$$

Подставляя (17) в (13), (14), с учетом (18), получим

$$
\begin{gathered}
R_{s r r}+\frac{\bar{\lambda}_{n}}{r^{2}} R_{s}+\mu_{s, n} R_{s}=0,0<r<1, \\
R_{s}(1)=0,\left|R_{s}(0)\right|<\infty, \\
T_{s t}+\mu_{s, n} g(t) T_{s}(t)=-a_{n s}^{k}(t), 0<t<\alpha, \\
T_{s}(\alpha)=0 .
\end{gathered}
$$

Ограниченным решением задачи (19), (20) является ([4])

$$
R_{s}(r)=\sqrt{r} J_{\nu}\left(\mu_{s, n} r\right)
$$

где $\nu=n+\frac{(m-2)}{2}, \mu_{s, n}-$ нули функций Бесселя первого рода $J_{\nu}(z), \mu=\mu_{s, n}^{2}$.

Решением задачи (21), (22)является

$$
T_{s, n}(t)=\left(\exp \left(-\mu_{s, n}^{2} \int_{0}^{t} g(\xi) d \xi\right)\right)\left(\int_{t}^{\alpha} a_{n s}^{k}(\xi)\left(\exp \mu_{s, n}^{2} \int_{0}^{\xi} g\left(\xi_{1}\right) d \xi_{1}\right) d \xi\right) .
$$

Подставляя (23) в (18) получим

$$
r^{-\frac{1}{2}} f_{n}^{k}(r, t)=\sum_{s=1}^{\infty} a_{n s}^{k}(t) J_{\nu}\left(\mu_{s, n} r\right), r^{-\frac{1}{2}} \tilde{\varphi}_{1 n}^{k}(r)=\sum_{s=1}^{\infty} b_{n s}^{k} J_{\nu}\left(\mu_{s, n} r\right), 0<r<1 .
$$

Ряды (25) - разложения в ряды Фурье-Бесселя [5],если

$$
\begin{gathered}
a_{n s}^{k}(t)=2\left[J_{\nu+1}\left(\mu_{s, n}\right)\right]^{-2} \int_{0}^{1} \sqrt{\xi} f_{n}^{k}(\xi, t) J_{\nu}\left(\mu_{s, n} \xi\right) d \xi, \\
b_{n s}^{k}=2\left[J_{\nu+1}\left(\mu_{s, n}\right)\right]^{-2} \int_{0}^{1} \sqrt{\xi} \tilde{\varphi}_{1 n}^{k}(\xi) J_{\nu}\left(\mu_{s, n} \xi\right) d \xi,
\end{gathered}
$$

$\mu_{s, n}, s=1,2, \ldots$ - положительные нули функций Бесселя $J_{\nu}(z)$, расположенные в порядке возрастания их величины.

Из (17), (23), (24) получим решение задачи (13), (14)

$$
v_{1 n}^{k}(r, t)=\sum_{s=1}^{\infty} \sqrt{r} T_{s}(t) J_{\nu}\left(\mu_{s, n} r\right)
$$

где $a_{n s}^{k}(t)$ определяется из $(26)$.

Далее, подставляя (17) в (15), (16), с учетом (18), будем иметь задачу

$$
T_{s t}+\mu_{s, n}^{2} g(t) T_{s}=0,0<t<\alpha, T_{s}(\alpha)=b_{n s}^{k},
$$

решением которого является

$$
T_{s, n}(t)=b_{n s} \exp \left(-\mu_{s, n}^{2} \int_{t}^{\alpha} g(\xi) d \xi\right)
$$


Из (23), (29) получим

$$
v_{2 n}^{k}(r, t)=\sum_{s=1}^{\infty} b_{n s}^{k} \sqrt{r}\left(\exp \mu_{s, n}^{2} \int_{t}^{\alpha} g(\xi) d \xi\right) J_{\nu}\left(\mu_{s, n} r\right),
$$

где $b_{n s}^{k}$ находится из (27).

Следовательно, единственным решением задачи (1), (2) в области $\Omega_{\alpha}$ является функция

$$
u(r, \theta, t)=\sum_{n=0}^{\infty} \sum_{k=1}^{k_{n}}\left\{\psi_{2 n}^{k}(t)+r^{\frac{(1-m)}{2}}\left[v_{1 n}^{k}(r, t)+v_{2 n}^{k}(r, t)\right]\right\} Y_{n, m}^{k}(\theta),
$$

где $v_{1 n}^{k}(r, t), v_{2 n}^{k}(r, t)$ определяются из $(28)$ и (30).

Учитывая формулу $([5]) 2 J_{\nu}^{\prime}(z)=J_{\nu-1}(z)-J_{\nu+1}(z)$, оценки $([6,3])$

$$
\begin{gathered}
J_{\nu}(z)=\sqrt{\frac{2}{\pi z}} \cos \left(z-\frac{\pi}{2} \nu-\frac{\pi}{4}\right)+0\left(\frac{1}{z^{3 / 2}}\right), \nu \geqslant 0, \\
\left|k_{n}\right| \leqslant c_{1} n^{m-2},\left|\frac{\partial^{q}}{\partial \theta_{j}^{q}} Y_{n, m}^{k}(\theta)\right| \leqslant c_{2} n^{\frac{m}{2}-1+q}, j=\overline{1, m-1}, q=0,1, \ldots,
\end{gathered}
$$

а также леммы, ограничения на заданные функции $\psi_{1}(t, \theta), \varphi_{1}(r, \theta)$, как в $[7,8]$, можно доказать, что полученное решение $(31)$ принадлежит классу $C\left(\bar{\Omega}_{\alpha}\right) \cap C^{2}\left(\Omega_{\alpha}\right)$.

Далее, из (28), (30), (31) $t \rightarrow+0$ при имеем

$$
\begin{gathered}
u(r, \theta, t)=\tau(r, \theta)=\sum_{n=0}^{\infty} \sum_{k=1}^{k_{n}} \tau_{n}^{k}(r) Y_{n, m}^{k}(\theta) \\
\tau_{n}^{k}(r)=\psi_{1 n}^{k}(0)+\sum_{s=1}^{\infty} r^{\frac{(2-m)}{2}}\left[\int_{0}^{\alpha} a_{n s}^{k}(\xi)\left(\exp \mu_{s, n}^{2} \int_{0}^{\xi} g\left(\xi_{1}\right) d \xi_{1}\right) d \xi+\right. \\
\left.+b_{n s}^{k} \exp \left(\mu_{s, n}^{2} \int_{0}^{\alpha} g(\xi) d \xi\right)\right] J_{n+\frac{(m-2)}{2}}\left(\mu_{s, n} r\right) .
\end{gathered}
$$

Из (26)-(28), (30), а также из лемм вытекает, что $\tau(r, \theta) \in W_{2}^{l}(S), l>\frac{3 m}{2}$.

Таким образом, учитывая краевые условия (3) и (32), мы приходим в области $\Omega_{\beta}$ к задаче Дирихле для вырождающихся многомерных эллиптических уравнений

с данными

$$
p(t) \Delta_{x} u+u_{t t}=0
$$

$$
\left.u\right|_{S}=\tau(r, \theta),\left.\quad u\right|_{\Gamma_{\beta}}=\psi_{2}(t, \theta),\left.u\right|_{\sigma_{\beta}}=\varphi_{2}(r, \theta),
$$

которое имеет единственное решение в классе $C\left(\bar{\Omega}_{\beta}\right) \cap C^{2}\left(\Omega_{\beta}\right)$ [8].

В [8] приводится явный вид решения задачи $(33),(34)$, поэтому можно записать представления решения и для задачи 1.

Теорема доказано.

Отметим, что приведенная теорема анонсировано в [9].

\section{Литература}

[1] Фикера Г. К единой теории краевых задач для эллиптико-параболических уравнений второго порядка: Сб. переводов. Математика. 1963. Т. 7. № 6. С. 99-121.

[2] Олейник О.А., Радкевич Е.В. Уравнения с неотрицательной характерической формой. М.: Изд-во Моск. ун-та, 2010. $360 \mathrm{c}$.

[3] Михлин С.Г. Многомерные сингулярные интегралы и интегральные уравнения. М.: Физматгиз, 1962. 254 с.

[4] Камке Э. Справочник по обыкновенным дифференциальным уравнениям. М.: Наука, 1965. 703 с.

[5] Бейтмен Г., Эрдейи А. Высшие трансцендентные функции. Т. 2. М.: Наука, 1974. 297 с.

[6] Тихонов А.Н., Самарский А.А. Уравнения математической физики. М: Наука, 1966. 724 с.

[7] Алдашев С.А. Краевые задачи для многомерных гиперболических и смешанных уравнений. Алматы: Гылым, 1994. $170 \mathrm{c}$.

[8] Алдашев С.А. Корректность задачи Дирихле в цилиндрической области для вырождающихся многомерных эллиптических уравнений // Матем. заметки. 2013. Т. 94. Вып. 6. С. 936-939.

[9] Алдашев С.А. Задача Дирихле в цилиндрической области для вырождающихся многомерных эллиптико-параболических уравнений // Тез. докладов межд. научной конференции "Актуальные проблемы теории уравнений в частных производных". М.: МГУ. 2016. С. 14. 


\section{References}

[1] Fiker G. K edinoi teorii kraevykh zadach dlia elliptiko-parabolicheskikh uravnenii vtorogo poriadka: Sb. perevodov [On a unified theory of boundary-value problems for second-order elliptic-parabolic equations: collection of translations]. Matematika [Mathematics], 1963, Vol. 7, no. 6, pp. 99-121 [in Russian].

[2] Oleinik O.A., Radkevich E.V. Uravneniia s neotritsatel'noi kharaktericheskoi formoi [Equations with a nonnegative characteristic form]. M.: Izd-vo Mosk. un-ta, 2010, 360 p. [in Russian].

[3] Mikhlin S.G. Mnogomernye singuliarnye integraly i integral'nye uravneniia [Multidimensional singular integrals and integral equations]. M.: Fizmatgiz, 1962, 254 p. [in Russian].

[4] Kamke E. Spravochnik po obyknovennym differentsial'nym uravneniiam [Handbook of ordinary differential equations]. M.: Nauka, 1965, 703 p. [in Russian].

[5] Bateman G., Erdei A. Vysshie transtsendentnye funktsii. T. 2 [Higher transcendental functions. Vol. 2]. M.: Nauka, 1974, 297 p. [in Russian].

[6] Tikhonov A.N., Samarskiy A.A. Uravneniia matematicheskoi fiziki [Equations of mathematical physics]. M: Nauka, 1966, 724 p. [in Russian].

[7] Aldashev S.A. Kraevye zadachi dlia mnogomernykh giperbolicheskikh i smeshannykh uravnenii [Boundary value problems for multidimensional hyperbolic and mixed equations]. Almaty: Gylym, 1994, 170 p. [in Russian].

[8] Aldashev S.A. Korrektnost' zadachi Dirikhle v tsilindricheskoi oblasti dlia vyrozhdaiushchikhsia mnogomernykh ellipticheskikh uravnenii [Correctness of the Dirichlet problem in a cylindrical domain for degenerate multidimensional elliptic equations]. [Mathematical Notes], 2013, Vol. 94, Issue 6, pp. 936-939 [in Russian].

[9] Aldashev S.A. Zadacha Dirikhle $v$ tsilindricheskoi oblasti dlia vyrozhdaiushchikhsia mnogomernykh elliptikoparabolicheskikh uravnenii [The Dirichlet problem in a cylindrical domain for degenerate multidimensional ellipticparabolic equations]. In: Tez. dokladov mezhd. nauchnoi konferentsii "Aktual'nye problemy teorii uravnenii $v$ chastnykh proizvodnykh" [Abstracts of the reports of the international scientific conference "Actual problems of the theory of partial differential equations"]. M.: MGU, 2016, p. 14 [in Russian].

\section{S.A. Aldashev ${ }^{2}$}

\section{THE CORRECTNESS OF THE DIRICHLET PROBLEM IN A CYLINDRICAL DOMAIN FOR DEGENERATE MULTIDIMENSIONAL ELLIPTIC-PARABOLIC EQUATIONS}

The correctness of boundary value problems on the plane for elliptic equations by the method of the theory of analytic functions of a complex variable has been well studied. When investigating similar questions, when the number of independent variables is greater than two, problems of a fundamental nature arise. A very attractive and convenient method of singular integral equations loses their validity due to the absence of any full theory of multidimensional singular integral equations. Boundary value problems for second-order elliptic equations in domains with edges have been studied in detail. In the author's papers explicit forms of classical solutions of Dirichlet problems in cylindrical domains for multidimensional elliptic equations are found. In this paper we use the method proposed in the author's works, we show the unique solvability and obtain an explicit form of classical solution of the Dirichlet problem in a cylindrical domain for degenerate multidimensional elliptico-parabolic equations.

Key words: correctness, multidimensional equations, degeneration, Dirichlet problem, cylindrical domain, spherical functions, orthogonality, Bessel function.

Статья поступила в редакцию $29 / I X / 2017$.

The article received $29 / I X / 2017$.

\footnotetext{
${ }^{2}$ Aldashev Serik Aimurzaevich (aldash51@mail.ru), Department of Mathematics and Mathematical Modeling, Institute of Mathematics and Mathematical Modeling, 125, Pushkin street, Almaty, 050010, Republic of Kazakhstan.
} 